\title{
ILMI Approach to Static Output Feedback Fuzzy Control for Synchroni- zation of Hyperchaotic Systems via T-S Models
}

\author{
Minxiu Yan ${ }^{1, *}$, Xiaofan Zheng ${ }^{1}$ and Zhuyin Xue ${ }^{2}$ \\ ${ }^{1}$ College of Information Engineering, Shenyang University of Chemical Technology, Shenyang, Liaoning, China, 110142; \\ ${ }^{2}$ College of Information Science and Engineering, Northeastern University, Shenyang, Liaoning, China, 110819
}

\begin{abstract}
A static output feedback fuzzy (SOFF) control approach is proposed in order to deal with the problem of synchronization of two identical hyperchaotic systems. The T-S fuzzy model with its small number of fuzzy IF-THEN rules is employed to represent many typical nonlinear hyperchaotic systems. Based on the T-S fuzzy hyperchaotic models and Lyapunov stability theory, a necessary condition for the existence of robust SOFF controllers is presented in terms of a set of matrix inequalities, which guarantees the stable synchronization of two different hyperchaotic systems. An iterative linear matrix inequality (ILMI) algorithm is proposed to compute the feedback gains of the suboptimal SOFF controller. Finally, numerical examples are given to demonstrate the validity and effectiveness of the proposed fuzzy modeling and synchronization scheme of the hyper chaotic systems.
\end{abstract}

Keywords: Hyperchaotic systems, iterative linear matrix inequality (ILMI), robust synchronization, static output feedback fuzzy controller, T-S fuzzy Models.

\section{INTRODUCTION}

Chaos is a very interesting nonlinear phenomenon mainly due to its complex dynamics such as, it is highly sensitive to initial conditions, fractal properties of the motion in phase space and so on. Since the pioneering work of Carroll and Pecora [1], synchronization of two chaotic dynamical systems have been paid increasing attention due to its powerful applications in information processing, secure communication, power converters, chemical reactions, biological systems etc. The control of chaos synchronization has attracted a lot of interest by variety of its systems including physical, chemical, biological and ecological systems. Many effective technologies have been developed such as sliding mode control method [2, 3], active control method [4, 5], adaptive control method $[6,7]$ and output feedback control method $[8$, 9] etc.

In the recent past, the state or output feedback control methods for synchronization of chaotic systems have been widely studied. In [10], a sufficient condition was proposed for chaos synchronization by the linear state feedback approach based on the Lyapunov stability theory in control theory. In [11], a simple linear feedback controller was proposed to make the states of two identical chaotic systems asymptotically synchronized. In [12], the robust control on the synchronization of a hyper-chaotic system with disturbance input was investigated. In [13], a static output feedback controller was proposed to guarantee robust synchronization between the master and slave systems based on linear matrix Inequality (LMI) and Lyapunov theory.

\footnotetext{
*Address correspondence to this authors at College of Information Engineering, Shenyang University of Chemical Technology, Liaoning, 110142, P.R. China; Tel: 13940440632; E-mail: cocoymx@sohu.com
}

The static output feedback (SOF) problem is one of the most researched problems in the control theory and its applications $[14,15]$. The SOF controller is less expensive to implement and more reliable in practice. On the other hand, many problems involving the synthesizing dynamic controller can be formulated as static output feedback control problems involving augmented plants. However, the static output feedback methods for synchronization of chaotic systems have limitations. The methods must assume the Lipschitz conditions of nonlinear terms but it is difficult to compute the parameters the Lipschitz conditions. To overcome this drawback, the control and synchronization chaotic systems uses fuzzy technique modeling and their stability analysis have been investigated extensively. Fuzzy technique has been widely and successfully used in nonlinear system modeling and control for more than two decades. The wellknown T-S fuzzy model $[16,17]$ is recognized as a popular and powerful tool in approximating a complex nonlinear system because of its simple structure with local dynamics. Lian et al [18] presented a synthesis approach for synchronizing the chaotic systems based on T-S fuzzy model. In their work, the parameters of controller are found by solving LMI problems. If it is possible to find appropriate matrices satisfying the LMI, then a stabilizing feedback gain exists. However, to the best knowledge of the authors, there is an infeasible LMI in many cases.

In this paper, a SOFF control approach is proposed for synchronizing two identical nonlinear hyperchaotic systems by employing the T-S fuzzy model. The T-S fuzzy models are employed to represent many typical nonlinear hyperchaotic systems exactly $[19,20]$. Based on the T-S fuzzy hyperchaotic models and Lyapunov stability theory, a necessary condition for the existence of robust SOF controller is presented in terms of a set of linear matrix inequalities (LMI), 
which guarantees the stable synchronization of two different hyperchaotic systems. On this basis, an improved iterative linear matrix inequality (ILMI) algorithm [21, 22] is proposed to compute the feedback gain of the suboptimal SOF controller. Finally, numerical examples are given to demonstrate the validity and effectiveness of the proposed fuzzy modeling and synchronization scheme of hyperchaotic systems.

\section{T-S FUZZY MODEL OF HYPERCHAOTIC SYN- CHRONIZED SYSTEMS}

\subsection{T-S Fuzzy Model}

In modern control theory, it is important to select an appropriate model to represent a nonlinear system.

As an expression of model, considering a class of nonlinear system represented by T-S fuzzy model as stated as follows:

Fuzzy Rule $i$ : IF $\omega_{1}(t)$ is $M_{i 1}$ and $\ldots \omega_{s}(t)$ is $M_{i s}$ THEN

$$
\begin{aligned}
& \dot{x}(t)=A_{i} x(t)+B u(t)+\delta \\
& y(t)=C x(t)
\end{aligned}
$$

where $i=1,2,3, \ldots r$ ( $r$ is the number of fuzzy rules), $x(t) \in R^{n}$ is the state vector, $u(t) \in R^{m}$ denotes the input vector, respectively, $A_{i} \in R^{n \times n}$ and $B \in R^{n \times m}$ are known as system matrix and input matrix dimensions, $\delta \in R^{n}$ is a constant vector and denotes a bias term which is generated by the fuzzy modeling procedure, $\omega_{1}(t), \ldots \omega_{s}(t) .(s$ is the number of premise variables)are premise variables, $M_{i j}(i=1,2 \ldots, r, j=1,2 \ldots, s)$ is a fuzzy set which is characterized by a membership function.

Taking a standard fuzzy inference method (using a singleton fuzzier, product fuzzy inference and center average defuzzifier), the final continuous-time fuzzy T-S system is inferred as follows:

$$
\dot{x}(t)=\frac{\sum_{i=1}^{r} v_{i}(\omega(t))\left[A_{i} x(t)+B u(t)+\delta\right]}{\sum_{i=1}^{r} v_{i}(\omega(t))}
$$

$y(t)=C x(t)$

where $v_{i}(\omega(t))=\prod_{j=1}^{s} M_{i j}\left(\omega_{j}(t)\right), v_{i}(\omega(t))(i=1,2 \ldots, r)$ is the membership function of the system with respect to the fuzzy rule $i$, and it satisfies:

$$
\sum_{i=1}^{r} v_{i}(\omega(t))>0 \text { And } v_{i}(\omega(t)) \geq 0 \text {. }
$$

The T-S system is considered as follows:

$$
\begin{aligned}
& \dot{x}(t)=\sum_{i=1}^{r} \mu_{i}(\omega(t))\left[A_{i} x(t)+B u(t)+\delta\right] \\
& y(t)=C x(t)
\end{aligned}
$$

where $\mu_{i}(\omega(t))=v_{i}(\omega(t)) / \sum_{i=1}^{r} v_{i}(\omega(t)) \cdot \mu_{i}$ Can be regarded as the normalized weight of each IF-THEN rule and it satisfies $\mu_{i}(\omega(t)) \geq 0$ and $\sum_{i=1}^{r} \mu_{i}(\omega(t))=1$.

\subsection{T-S Fuzzy Synchronization Model of the Hyper- Chaotic Systems}

Note that most of the hyperchaotic systems can be expressed as follows:

$\dot{x}(t)=A x(t)+f(x(t))$

where $x(t) \in R^{n}$ is the $\mathrm{n}$-dimensional state vector of the system, $A \in R^{n \times n}$ denotes the linear part of the system dynamics and $f(x(t))$ represents the nonlinear parts of the system.

According to the boundedness of the hyperchaotic systems, we can find that $x_{i}(t) \in\left[c_{i}-d_{i}, c_{i}+d_{i}\right]$, where $d_{i}>0, c_{i}$ and $d_{i}$ are obtained by the numerical simulation. We can assume that there is a common factor in the nonlinear parts and choose the common factor as the premise variable of T-S fuzzy rules. For example, in the following Lorenz hyperchaotic system, the nonlinear parts include common factor $x_{1}$. Therefore we can choose $x_{1}$ as the premise variable.

$\left\{\begin{array}{l}\dot{x}_{1}=a\left(x_{2}-x_{1}\right)+x_{4} \\ \dot{x}_{2}=-x_{1} x_{3}+b x_{1}-x_{2} \\ \dot{x}_{3}=x_{1} x_{2}-c x_{3} \\ \dot{x}_{4}=-x_{1} x_{3}+d x_{4}\end{array}\right.$

The membership functions are chosen as

$v_{1}\left(x_{1}(t)\right)=\frac{1}{2}\left(1-\frac{c_{1}-x_{1}(t)}{d_{1}}\right), v_{2}\left(x_{1}(t)\right)=\frac{1}{2}\left(1+\frac{c_{1}-x_{1}(t)}{d_{1}}\right)$

We can obtain the following T-S model system:

$\dot{x}(t)=\frac{\sum_{i=1}^{2} v_{i}(\omega(t))\left[A_{i} x(t)+B u(t)+\delta\right]}{\sum_{i=1}^{2} v_{i}(\omega(t))}$

For the Lorenz hyperchaotic system, the $A_{1}, A_{2}$ are chosen as follow:

$A_{1}=\left[\begin{array}{cccc}-a & a & 0 & 1 \\ b & 1 & -\left(c_{1}+d_{1}\right) & 0 \\ 0 & \left(c_{1}+d_{1}\right) & -c & 0 \\ 0 & 0 & -\left(c_{1}+d_{1}\right) & d\end{array}\right]$
$A_{2}=\left[\begin{array}{cccc}-a & a & 0 & 1 \\ b & 1 & -\left(c_{1}-d_{1}\right) & 0 \\ 0 & \left(c_{1}-d_{1}\right) & -c & 0 \\ 0 & 0 & -\left(c_{1}-d_{1}\right) & d\end{array}\right]$ 
Consider the nonlinear hyperchaotic system as master system:

$\dot{x}=A x+f(x)$

$y(t)=C x(t)$

And another hyperchaotic system as slave system:

$\dot{\hat{x}}=A \hat{x}+f(\hat{x})+u(t)$

$\hat{y}(t)=C \hat{x}(t)$

where $x, \hat{x} \in R^{n}$ are the $\mathrm{n}$-dimensional state vectors of the system, $\mathrm{A} \in R^{n \times n}$ denotes the linear part of the system dynamics and $f(x), f(\hat{x}): R^{n} \rightarrow R^{n}$ represent the nonlinear parts of the system, $u(t)$ is the output vector of the controller.

The master system is inferred as follows:

$$
\begin{aligned}
\dot{x}(t) & =\sum_{i=1}^{r} \mu_{i}(\omega(t)) A_{i} x(t) \\
y(t) & =C x(t)
\end{aligned}
$$

The slave system is inferred as follows:

$$
\begin{aligned}
\dot{\hat{x}}(t) & =\sum_{i=1}^{r} \mu_{i}(\omega(t))\left[A_{i} \hat{x}(t)+B u(t)\right] \\
\hat{y}(t) & =C \hat{x}(t)
\end{aligned}
$$

where $\mu_{i}(\omega(t))=\frac{v_{i}(\omega(t))}{\sum_{i=1}^{2} v_{i}(\omega(t))}$

The synchronization error system between (11) and (12) can be written as follows:

$e=\hat{x}-x$

The synchronization error of the dynamics system is inferred as follows:

$$
\begin{aligned}
\dot{e} & =\sum_{i=1}^{r} \mu_{i}(\omega(t)) A_{i} \hat{x}(t)+B u(t)-\sum_{i=1}^{r} \mu_{i}(\omega(t)) A_{i} x(t) \\
& =\sum_{i=1}^{r} \mu_{i}(\omega(t)) A_{i} e(t)+B u(t)
\end{aligned}
$$

The objective of this paper is to design a feedback control law such that $\lim _{t \rightarrow \infty}\|e(t)\|=0$.

\section{STATIC OUTPUT FEEDBACK CONTROLLER DE- SIGN AND ANALYSIS}

In this section, we will investigate the synchronization controller of two hyperchaotic systems based on the T-S fuzzy models.

Lemma 1. For a given symmetric matrix $S=\left[\begin{array}{ll}S_{11} & S_{12} \\ S_{21} & S_{22}\end{array}\right]$, where $S_{11}{ }^{\mathrm{T}}=S_{11}, \quad S_{12}{ }^{\mathrm{T}}=S_{21}, \quad S_{22}{ }^{\mathrm{T}}=S_{22}$, the condition $S<0$ is equivalent to $S_{22}<0$ and $S_{11}-S_{12} S_{22}{ }^{-1} S_{12}{ }^{\mathrm{T}}<0$.

Theorem 1. For given $X_{i}>0$, if there exist positivedefinite symmetric matrix $P_{i}^{\mathrm{T}}=P_{i}$ and any matrix $K_{i}$ satisfying the following LMI:

$$
\begin{array}{r}
{\left[\begin{array}{l}
A_{i}^{\mathrm{T}} P+P A_{i}-X_{i} B B^{\mathrm{T}} P_{i}-P_{i} B B^{\mathrm{T}} X_{i}+X_{i} B B^{\mathrm{T}} X_{i} \\
B^{\mathrm{T}} P_{i}+K_{i} C
\end{array}\right]<0} \\
\left.\quad\left(B^{\mathrm{T}} P_{i}+K_{i} C\right)^{\mathrm{T}}\right]< \\
\quad-I
\end{array}
$$

Then dynamics system (14) is stabilizable and the controller is given by

$u(t)=\sum_{i=1}^{r} \mu_{i}(\omega(t))\left(K_{i} C(\hat{x}(t)-x(t))\right)$

Proof. The controller is described by the following rules:

Fuzzy Rule $i$ : IF $\omega_{1}(t)$ is $M_{j 1}$ and... $\omega_{s}(t)$ Is $M_{i s}$ Then

$u(t)=K_{i}(\hat{y}(t)-y(t))=K_{i} C(\hat{x}(t)-x(t))$

Where $K_{i} \in R^{n \times m}$ is the feedback gain matrix of the controller for the fuzzy rule $i$. The fuzzy controller can be inferred as Eq.(16). The dynamics of the synchronization error system can be written as follows:

$$
\begin{aligned}
\dot{e} & =\sum_{i=1}^{r} \mu_{i}(\omega(t))\left[A_{i} e(t)+\left(B K_{j} C(\hat{x}(t)-x(t))\right)\right] \\
& =\sum_{i=1}^{r} \mu_{i}(\omega(t))\left(A_{i}+B K_{j} C\right) e(t)
\end{aligned}
$$

Choose the Lyapunov function as $V=e(t)^{\mathrm{T}} P e(t)$, where $P^{\mathrm{T}}=P>0$.

Taking derivative of the Lyapunov function candidate with respect to time, one has

$$
\begin{aligned}
\dot{V} & =\dot{e}(t)^{\mathrm{T}} P e(t)+e(t)^{\mathrm{T}} P \dot{e}(t) \\
& =\sum_{i=1}^{r} \mu_{i}(\omega(t)) \dot{e}(t)^{T}\left[\left(A_{i}+B K_{j} C\right)^{\mathrm{T}} P+P\left(A_{i}+B K_{j} C\right)\right] e(t) \\
& \leq \sum_{i=1}^{r} \mu_{i}(\omega(t)) \dot{e}(t)^{T}\left[\left(A_{i}+B K_{j} C\right)^{\mathrm{T}} P+P\left(A_{i}+B K_{j} C\right)\right. \\
& \left.+C^{\mathrm{T}} K_{j}^{\mathrm{T}} K_{j} C\right] e(t) \\
& =\sum_{i=1}^{r} \mu_{i}(\omega(t)) \dot{e}(t)^{T}\left[A_{i}^{\mathrm{T}} P+P A_{i}-P_{i} B B^{\mathrm{T}} P_{i}\right. \\
& \left.+\left(B^{\mathrm{T}} P_{i}+K_{i} C\right)^{\mathrm{T}}\left(B^{\mathrm{T}} P_{i}+K_{i} C\right)\right] e(t)
\end{aligned}
$$

There exists a negative sign in the $-P_{i} B B^{\mathrm{T}} P_{i}$ term. The inequality cannot be simplified to LMI. Therefore we introduce an additional positive-definite matrix variable $X_{i}$.

Obviously, $\left(X_{i}-P_{i}\right)^{\mathrm{T}} B B^{\mathrm{T}}\left(X_{i}-P_{i}\right) \geq 0, \quad$ where $X_{i}, P_{i}$ are the same dimension matrix. We obtain 
$-X_{i} B B^{\mathrm{T}} P_{i}-P_{i} B B^{\mathrm{T}} X_{i}+X_{i} B B^{\mathrm{T}} X_{i} \geq-P_{i} B B^{\mathrm{T}} P_{i}$

Then we obtain

$$
\begin{aligned}
\dot{V} & \leq \sum_{i=1}^{r} \mu_{i}(\omega(t)) \dot{e}(t)^{T}\left[A_{i}^{\mathrm{T}} P+P A_{i}-X_{i} B B^{\mathrm{T}} P_{i}-P_{i} B B^{\mathrm{T}} X_{i}\right. \\
& \left.+X_{i} B B^{\mathrm{T}} X_{i}+\left(B^{\mathrm{T}} P_{i}+K_{i} C\right)^{\mathrm{T}}\left(B^{\mathrm{T}} P_{i}+K_{i} C\right)\right] e(t)
\end{aligned}
$$

If the following matrix inequality is satisfied:

$A_{i}^{\mathrm{T}} P+P A_{i}-X_{i} B B^{\mathrm{T}} P_{i}-P_{i} B B^{\mathrm{T}} X_{i}+X_{i} B B^{\mathrm{T}} X_{i}$

$+\left(B^{\mathrm{T}} P_{i}+K_{i} C\right)^{\mathrm{T}}\left(B^{\mathrm{T}} P_{i}+K_{i} C\right) \leq 0$

We can know

$V=e(t)^{\mathrm{T}} P e(t)>0$ And $\dot{V} \leq 0$

Based on Lyapunov's theory, if there exists positivedefinite symmetric matrix $P_{i}^{\mathrm{T}}=P_{i}$ and any matrix $K_{i}$, dynamics system (14) is stabilizable.

Applying the Schur complement, the inequality (22) can be equivalent to

$$
\begin{gathered}
{\left[\begin{array}{ll}
A_{i}^{\mathrm{T}} P+P A_{i}-X_{i} B B^{\mathrm{T}} P_{i}-P_{i} B B^{\mathrm{T}} X_{i}+X_{i} B B^{\mathrm{T}} X_{i} \\
B^{\mathrm{T}} P_{i}+K_{i} C
\end{array}\right]<0} \\
\\
\quad\left(B^{\mathrm{T}} P_{i}+K_{i} C\right)^{\mathrm{T}} \\
-I
\end{gathered}
$$

If $X_{i}$ is fixed in Eq.(24), then it reduces to LMI problem for the unknowns $P_{i}$ and $K_{i}$.

Lemma 2. The dynamics system (14) is $\alpha / 2$-stabilizable via static output feedback if and only if there exist two matrices $P_{i}$ and $K_{i}$ satisfying the following matrix inequality:

$$
\begin{aligned}
& A_{i}^{\mathrm{T}} P+P A_{i}-X_{i} B B^{\mathrm{T}} P_{i}-P_{i} B B^{\mathrm{T}} X_{i}+X_{i} B B^{\mathrm{T}} X_{i} \\
& +\left(B^{\mathrm{T}} P_{i}+K_{i} C\right)^{\mathrm{T}}\left(B^{\mathrm{T}} P_{i}+K_{i} C\right)-\alpha_{i} P_{i} \leq 0
\end{aligned}
$$

The closed-loop system matrices have eigenvalues on the left-hand side of the line $\mathrm{R}(\mathrm{s})=\alpha_{i}$ a in the complex s-plane. Based on the idea that all eigenvalues of closed-loop system matrices are shifted progressively towards the left-half-plane through the reduction of $\alpha_{i}$, thus the ILMI algorithm is introduced.

The dynamics system is in realization with $\left(A_{i}, B\right.$, and $\left.C\right)$. And the ILMI algorithm is expressed as follows:

Step 1. Choose $Q_{i}>0$ and solve $P$ for the Riccati equation $A^{\mathrm{T}}{ }_{i} P_{i}+P_{i} A_{i}-P_{i} B B^{\mathrm{T}} P_{i}+Q_{i}=0, P_{i}>0$. Set $j=1$ and $X_{i 1}=P$.

Step 2. Solve the following optimization problem for, $P_{i j}, K_{i}$ and $\alpha_{i j}$.

OP1. Minimize $\alpha_{i j}$ subject to the following LMI constraints

$$
\begin{gathered}
{\left[\begin{array}{c}
A_{i}^{\mathrm{T}} P_{i j}+P_{i} A_{i}-X_{i j} B B^{\mathrm{T}} P_{i j}-P_{i j} B B^{\mathrm{T}} X_{i j}+X_{i j} B B^{\mathrm{T}} X_{i j} \\
B^{\mathrm{T}} P_{i j}+K_{i} C
\end{array}\right]<0} \\
-\alpha_{i j} P_{i j}\left(B^{\mathrm{T}} P_{i j}+K_{i} C\right)^{\mathrm{T}} \\
-\mathrm{I} \\
P_{i j}=P_{i j}{ }^{\mathrm{T}}>0
\end{gathered}
$$

Denoted by $\alpha_{i j}{ }^{*}$ the minimized value of $\alpha_{i j}$.

Step 3. If $\alpha_{i j}{ }^{*}<0$, set $P_{i}=P_{i j}$ and $K_{i}$ is a stabilizing static output feedback gain. Stop.

Step 4. Solve the following optimization problem for $P_{i j}$ and $K_{i}$.

OP2. Minimize trace $P_{i j}$ subject to the above LMI constraints (26) with $\alpha_{i j}=\alpha_{i j}{ }^{*}$. Denote by $P_{i j}{ }^{*}$ as the optimal trace $P_{i j}$.

Step 5. If $\left\|X_{i j}-P_{i j}^{*}\right\|<\varepsilon$, where $\varepsilon$ is a prescribed tolerance, go to Step 6, else set $j=j+1$ and $X_{i j}=P_{i j-1}{ }^{*}$, then go to Step 2.

Step 6. The system may not be stabilizable via static output feedback. Stop

If the algorithm fails to arrive at a stabilizing solution, we may select another $Q$ and run the ILMI algorithm again.

\section{NUMERICAL SIMULATIONS}

In this section, the numerical simulation results of hyperchaotic systems are discussed and validate the effectiveness.

The hyperchaotic Liu system is described by

$\left\{\begin{array}{l}\dot{x}_{1}=a\left(x_{2}-x_{1}\right) \\ \dot{x}_{2}=b x_{1}-c x_{1} x_{3}+x_{4} \\ \dot{x}_{3}=-d x_{3}+g x_{1}^{2} \\ \dot{x}_{4}=-r x_{4}\end{array}\right.$

where $a=10, b=40, c=1, d=2.5, g=4, r=10.6$.

The attractors of Liu hyperchaotic system are shown in Figs. (1-4). The initial state is chosen as $x(0)=[2 ; 1 ; 5 ; 2]$.

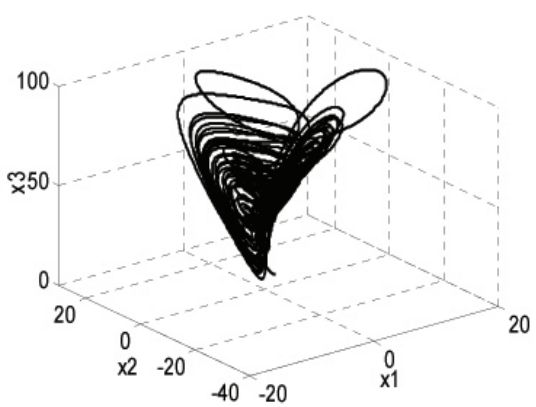

Fig. (1). Attractor of Liu hyperchaotic on x1-x2-x3. 


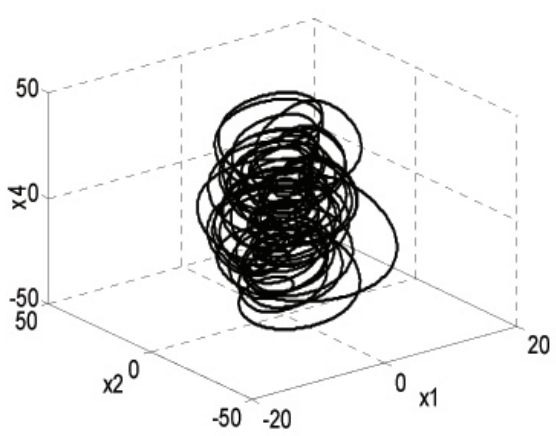

Fig. (2). Attractor of Liu hyperchaotic on $\mathrm{x} 1-\mathrm{x} 2-\mathrm{x} 4$.

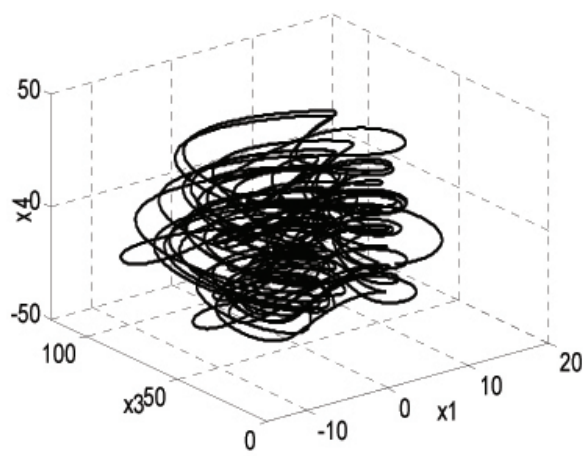

Fig. (3). Attractor of Liu hyperchaotic on x1-x3-x4.

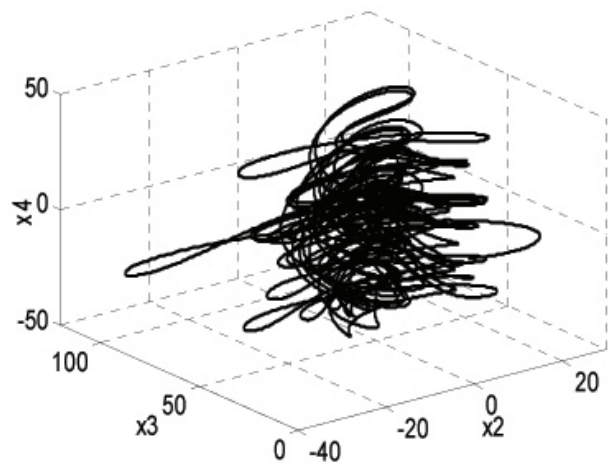

Fig. (4). Attractor of Liu hyperchaotic on $\mathrm{x} 2-\mathrm{x} 3-\mathrm{x} 4$.

From the simulation results we can get $x_{1} \in[1.2-18.7,1.2+18.7]$. Obviously, the nonlinear parts include a common factor $x_{1}(t)$. We can then derive the T-S fuzzy models of Liu hyperchaotic system as follows:
R1: IF $x_{1}(t)$ is $M_{1}$, THEN $\dot{x}(t)=A_{1} x(t)+\delta$,

R2: IF $x_{1}(t)$ is $M_{2}$, THEN $\dot{x}(t)=A_{2} x(t)+\delta$, where

$$
A_{1}=\left[\begin{array}{cccc}
-10 & 10 & 0 & 0 \\
40 & 0 & -19.9 & 1 \\
79.6 & 0 & -2.5 & 0 \\
-10.6 & 0 & 0 & 0
\end{array}\right] A_{2}=\left[\begin{array}{cccc}
-10 & 10 & 0 & 0 \\
40 & 0 & 17.4 & 1 \\
69.6 & 0 & -2.5 & 0 \\
-10.6 & 0 & 0 & 0
\end{array}\right] \delta=\left[\begin{array}{l}
0 \\
0 \\
0 \\
0
\end{array}\right]
$$

The membership functions are chosen as:

$$
\begin{aligned}
& M_{1}\left(x_{1}(t)\right)=\frac{1}{2}\left(1-\frac{1.2-x_{1}(t)}{18.7}\right) \\
& M_{2}\left(x_{1}(t)\right)=\frac{1}{2}\left(1+\frac{1.2-x_{1}(t)}{18.7}\right)
\end{aligned}
$$

Based on the above T-S fuzzy hyperchaotic models, we investigate the synchronization between Liu hyperchaotic systems. In simulation the initial values are assumed to be $\left(x_{1}(0), x_{2}(0), x_{3}(0), x_{4}(0)\right)=(2,1,5,2)$. In the numerical simulation, we use the following parameters:

$$
B=\left[\begin{array}{ll}
1 & 0 \\
0 & 1 \\
0 & 0 \\
0 & 0
\end{array}\right] C=\left[\begin{array}{llll}
1 & 0 & 0 & 0 \\
0 & 1 & 0 & 0
\end{array}\right]
$$

Choose $Q=I$, Theorem 1 works well using LMI toolbox in MATLAB. However, Theorem 1 does not lead to a feasible conclusion. Therefore, by applying ILMI algorithm to solve the corresponding LMI, We obtain a set of feasible solutions. Simulation results for Theorem 1 and ILMI algorithm studies are presented in Table $\mathbf{1}$.

We get the positive definite matrices $P_{1}$ and $P_{2}$ as follows:

\begin{tabular}{|c|c|c|c|c|}
\hline & \multirow{2}{*}{$\begin{array}{c}\text { LMI } \\
\text { Feasibility }\end{array}$} & \multicolumn{3}{|c|}{ ILMI } \\
\hline & & Feasibility & $\mathbf{K i}$ & Poles \\
\hline A1 & infeasible & feasible & $K_{1}=\left[\begin{array}{cc}-1810.5 & 1078.8 \\
114.9 & -135.2\end{array}\right]$ & $\begin{array}{c}-1915.7 \\
-21.2+23.6 \mathrm{i} \\
-21.2-23.6 \mathrm{i} \\
-0.1\end{array}$ \\
\hline A2 & infeasible & feasible & $K_{2}=\left[\begin{array}{cc}-1308.6 & 547.6 \\
9.9 & -37.1\end{array}\right]$ & $\begin{array}{c}-1339.2 \\
-34.6+21.4 \mathrm{i} \\
-34.6-21.4 \mathrm{i} \\
-1.5\end{array}$ \\
\hline
\end{tabular}

$$
\begin{aligned}
& P_{1}=\left[\begin{array}{cccc}
1679.7 & -998.2 & 0.6 & 0 \\
-998.2 & 680.6 & 3.4 & -3 \\
6 & 3.4 & 34.7 & -1 \\
0 & -3 & -1 & 11.7
\end{array}\right] \\
& P_{2}=\left[\begin{array}{cccc}
950.76 & -395.84 & -4.23 & -0.14 \\
-395.84 & 206.29 & 1.51 & -0.14 \\
-4.23 & 1.51 & 22.92 & 0.014 \\
-0.14 & -0.14 & 0.014 & 11.7
\end{array}\right]
\end{aligned}
$$

Table 1. Simulation results for the Theorem 1 and ILMI algorithm. 
Numerical simulations are carried out using the MATLAB software. The ode45 solver is used for solve synchronization problem.

Figs. (5-8) show the synchronization errors. It can be seen that the slave system can trace the master system successfully when output of the proposed controller is in action after 10 s.

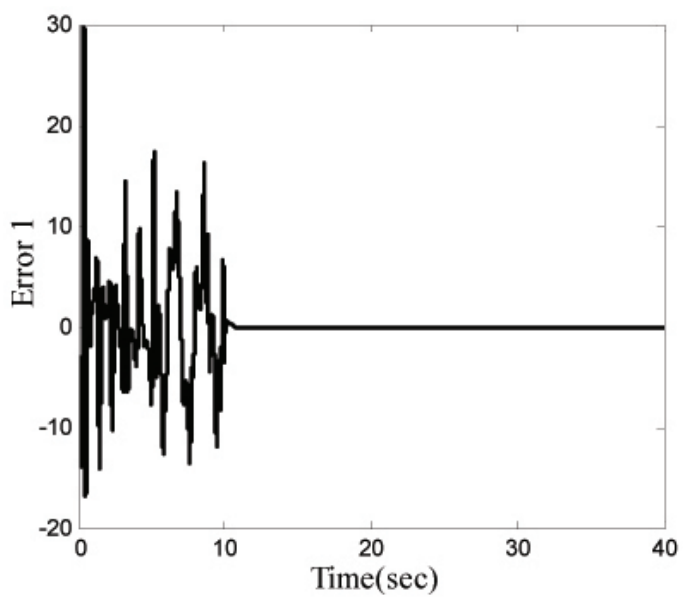

Fig. (5). Synchronization error 1.

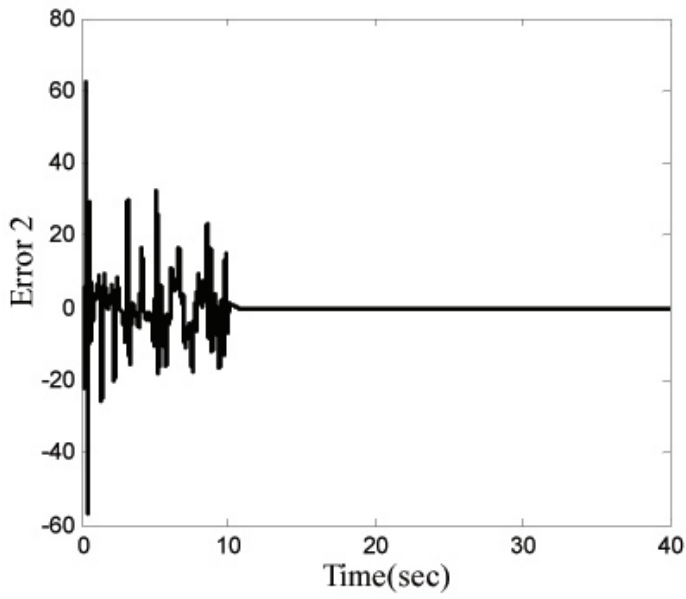

Fig. (6). Synchronization error 2.

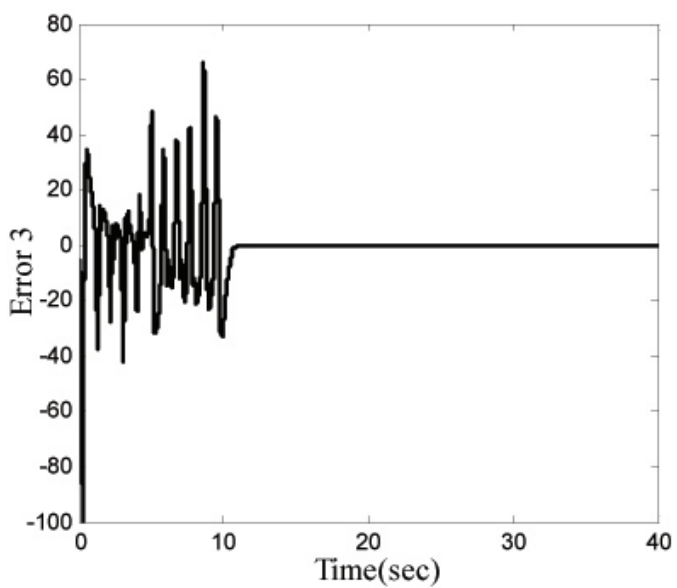

Fig. (7). Synchronization error 3.

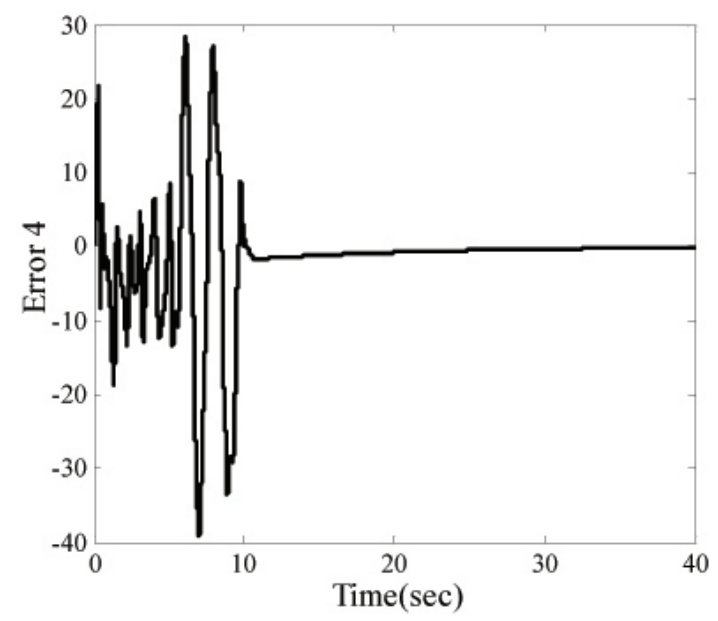

Fig. (8). Synchronization error 4.

\section{CURRENT \& FUTURE DEVELOPMENTS}

So far, the research of hyperchaotic systems is a hot spot in the research world. Compared with normal chaotic systems, hyperchaotic systems have more sensitivity to initial conditions and randomness which makes a secure communication system even more secured. Even if the information is intercepted, separating out the useful information is almost impossible. The static output feedback method for synchronization of hyperchaotic systems is less expensive to implement and more reliable in practice. However, the research on hyperchaotic systems is not mature enough. Many problems such as how to shorten the time synchronization and how to enhance the efficiency of operations etc. still need to be solved in the future.

\section{CONFLICT OF INTEREST}

The authors confirm that this article content has no conflict of interest.

\section{ACKNOWLEDGEMENTS}

This work is supported by the National Science and Technology Support Projects of China (No. 2012BAF09B 01).

\section{REFERENCES}

[1] L.M. Pecora, and T.L. Carroll, "Synchronization in chaotic systems," Physical review letters, vol. 64, pp. 821-824, 1990.

[2] J. J. Yan, Y. S. Yang, T. Y. Chiang, and C. Y. Chen, "Robust synchronization of unified chaotic systems via sliding mode control," Chaos, Solitons \& Fractals, vol.34, pp. 947-954, 2007.

[3] S. Vaidyanathan, "Sliding mode controller design for synchronization of Shimizu-Morioka chaotic systems," International Journal of Information, vol.1, pp. 20-29, 2011.

[4] M. T. Yassen, "Chaos synchronization between two different chaotic systems using active control," Chaos, Solitons \& Fractals, vol. 23, pp. 131-140, 2005.

[5] H. K. Chen, "Global chaos synchronization of new chaotic systems via nonlinear control," Chaos, Solitons \& Fractals, vol. 23, pp. 1245-1251, 2005.

[6] S. Chen, and J. Lü, "Synchronization of an uncertain unified chaotic system via adaptive control," Chaos, Solitons \& Fractals, vol. 14, pp. 643-647, 2002.

[7] X. F.Li, A. C. S. Leung, X. P. Han, X. J. Liu, and Y. D. Chu, 
"Complete (anti-) synchronization of chaotic systems with fully uncertain parameters by adaptive control," Nonlinear Dynamics, vol. 63, pp. 263-275, 2011.

[8] H. H. Chen, G. J. Sheu,Y. L. Lin, and C. S. Chen, "Chaos synchronization between two different chaotic systems via nonlinear feedback control, "Nonlinear Analysis: Theory, Methods \& Applications, vol. 70, pp. 4393-4401, 2009.

[9] C. K. Ahn, "T-S fuzzy Ho synchronization for chaotic systems via delayed output feedback control," Nonlinear Dynamics, vol. 59, pp. 535-543, 2010

[10] G. P. Jiang, and W. X. Zheng, "An LMI criterion for linear-statefeedback based chaos synchronization of a class of chaotic systems," Chaos, Solitons \& Fractals, vol. 26, pp. 437-443, 2005.

[11] S. Kuntanapreeda, "Chaos synchronization of unified chaotic systems via LMI," Physics Letters A, vol. 373-32, pp. 2837-2840, 2009.

[12] B. Wang, P. Shi, H. R. Karimi, Y. D. Song, and J. Wang, "Robust $\mathrm{H} \infty$ synchronization of a hyper-chaotic system with disturbance input," Nonlinear Analysis: Real World Applications, vol. 14, pp. 1487-1495, 2013.

[13] Y. Y. Hou, T. L. Liao, and J. J. Yan, "Hळ synchronization of chaotic systems using output feedback control design," Physica A: Statistical Mechanics and its Applications, vol. 379, pp. 81-89, 2007.

[14] V. L. Kharitonov, S. I. Niculescu, J. Moreno, and S. W. Michiel, "Static output feedback stabilization: Necessary conditions for multiple delay controllers," IEEE Transactions on Automatic Control, vol. 50, pp. 82-86, 2005.

[15] A. R. Mustapha, "Solvability of static output-feedback stabilization for LTI positive systems," Systems \& Control Letters, vol. 60, pp. 704-708, 2011.

[16] T. Takagi, and M. Sugeno, "Fuzzy identification of systems and its applications to modeling and control," IEEE Transactions on Systems, vol. 15-1, pp. 116-132, 1985.

[17] C. Lin, Q. G. Wang, and L. T. Heng, "Improvement on observerbased $\mathrm{H} \infty$ control for T-S fuzzy systems," Automatica, vol. 41, pp. 1651-1656, 2005.

[18] K. Y. Lian, T. S.Chiang, C. S. Chiu, and P. Liu, "Synthesis of fuzzy model-based designs to synchronization and secure communications for chaotic systems, "IEEE Transactions on Systems, vol. 31 pp. 66-83, 2001.

[19] H. Zhang, X. Liao, and J.Yu, "Fuzzy modeling and synchronization of hyperchaotic systems," Chaos, Solitons \& Fractals, vol. 26, pp. 835-843, 2005.

[20] Y. Zhao, T. Y. Zhang, D. S.Yang, and X. H. Zhang, "Fuzzy modeling and $\mathrm{H} \infty$ synchronization of different hyperchaotic systems via T-S models," Applied Mathematics \& Information Sciences, vol. 7 , pp. 193-200, 2013.

[21] H. N. Wu, "An ILMI approach to robust H2 static output feedback fuzzy control for uncertain discrete-time nonlinear systems," Automatica, vol. 44, pp. 2333-2339, 2008.

[22] Y. Y. Cao, J. Lam, and Y. X.Sun, "Static output feedback stabilization :an ILMI approach," Automatica, vol. 34, pp. 1641-1645, 1998.

Received: September 22, 2014

Revised: November 30, 2014

Accepted: December 02, 2014

(C) Yan et al.; Licensee Bentham Open.

This is an open access article licensed under the terms of the Creative Commons Attribution Non-Commercial License (http://creativecommons.org/licenses/by-nc/3.0/) which permits unrestricted, non-commercial use, distribution and reproduction in any medium, provided the work is properly cited. 